\section{Asociación entre el nivel de educación y la sobrevida después de presentar un infarto agudo de miocardio}

\section{Association between educational level and survival after acute myocardial infarction}

\section{Sr. Editor:}

En una reciente publicación Nazzal y col., evaluaron la asociación entre el nivel de educación y la sobrevida después de presentar un infarto agudo de miocardio (IAM) en una muestra de pacientes procedente del Registro Chileno de Infarto de Miocardio (GEMI) ${ }^{1}$. Los autores indican que un bajo nivel educacional se asocia con un mal pronóstico en pacientes con IAM; sin embargo, los autores no especifican el intervalo de edad de la población incluida en el estudio, esto es muy importante ya que se ha demostrado que el pronóstico de un paciente con IAM se ve influenciado por la edad y el sexo. Otro factor importante a considerar por los autores es el empleo de la clasificación internacional de enfermedades (CIE-10) como medida de resultado. Los autores no especifican si se emplearon casos incidentes o prevalentes de IAM y cuáles fueron los códigos que se emplearon para la definición de casos; por lo que el análisis no permite distinguir que tipo de causa de muerte se empleó como evento primario de estudio, y limita el estudio a la definición de evento primario como una suma de diferentes causas de fallecimiento y no se genera un sub-análisis de eventos individuales los cuales se pueden evaluar como eventos secundarios (por causa de fallecimiento no agrupada). Estos análisis permitirían conocer de una forma más precisa la relación entre el nivel de educación y las causas de muerte reportadas por los hospitales. La información proporcionada para categorizar el nivel de educación para la categoría de educación secundaria y básico es confusa, esto es importante debido a que los autores indican que sus resultados reafirman la necesidad de realizar estudios a futuro considerando más variables sociales y subrayan el impacto que este tipo de análisis tiene sobre las políticas de salud de los países de la región. Por ello, presentar una definición clara de cada nivel de educación permite la comparación de resultados entre varias zonas geográficas. Además al ser un estudio de cohorte; el cual muestra resultados analizados mediante hazard ratios, permite la realización futura de meta-análisis tanto para Chile como país, así como para los países de la región, lo que resalta la importancia de proporcionar definiciones claras. Más aun, el análisis de los resultados por sexo permitiría conocer si se obtienen los mismos resultados para ambos tipos de población; incluso para grupos de individuos con diferentes edades. Igland y col., mostraron en población Noruega que la tasa de incidencia de IAM es diferente en los hombres y las mujeres de diferentes grupos de edad (35-69 años y 70-94 años) ${ }^{2}$. No obstante, aunque el estudio muestra que el porcentaje de letalidad es diferente con respecto al nivel de educación, con una mayor letalidad en la población sin estudios, la Figura 1a muestra los porcentajes de mortalidad dentro del primer mes posterior a un IAM con respecto a la población total; mientras que la figura $1 \mathrm{~b}$ muestra los datos respecto a la mortalidad posterior a un mes de haber presentado un IAM en el resto de la población y sin embargo no se discute la influencia de los tamaños de cada sub-grupo dentro del análisis. Los datos indican que el 7,2\% de la población analizada presento un evento de mortalidad en el primer mes de seguimiento; aproximadamente el $16 \%$ dentro del grupo con estudios superiores, el 37\%, $41 \%$ y $6 \%$ para los grupos con estudios de nivel medio, básico y sin estudios; respectivamente, del total de la población con dicha característica $(n=262)$. Este análisis deja ver que la mayor prevalencia de letalidad se observa en los grupos con un nivel de estudios básico y medio, lo que contrasta con los porcentajes de la Figura 1a. Sin embargo, es muy interesante observa que a pesar de esta alta prevalencia en estos grupos, el análisis de riesgos muestra que el riesgo se incrementa en el grupo de sujetos sin estudios. Además, esta misma proporción se observó para los sujetos con una letalidad de más de un meses (letalidad a 30 días versus letalidad desde mes 2 a mes 55: superior $=16,41 \%$ vs $17,53 \%$; medio $=37,02 \%$ vs $37,89 \%$; básico $=40,84 \%$ vs $39,43 \%$ y ninguno $=5,72 \%$ vs $5,15 \%)$. Otro análisis interesante que no se discute en el artículo se observa en la Tabla 4 , donde se puede ver que el uso de medicamentos (estatinas, beta-bloqueadores, aspirina, inhibidores de la ECA o ARA II intrahospitalario) parecer ser un factor predictivo que mejora la sobrevida de los pacientes, tampoco se indica si el análisis de HR para estas variables contempla a los individuos tratados con monoterapia o hay combinación de medicamentos. Otra conclusión a la que llegan los autores es que aparentemente la sobrevida de un paciente se ve influida por el tipo de nosocomio donde se realiza su atención. No obstante a que se observa que existe un riesgo de 1,27 veces mayor de mortalidad al ser atendido en un hospital público, el intervalo de confianza y el valor de probabilidad son limítrofes, lo cual hace sentido con la alta proporción de personas incluidas dentro del análisis procedentes de un hospital público $(2.564$ sujetos con antecedente de hospitalización en un nosocomio público, 70,90\%; 788 sujetos hospitalizados en un nosocomio privado, $21,67 \%$ y 270 sujetos hospitalizados en un centro de las fuerzas armadas, 7,43\%), por lo que esta asociación depende más del tamaño de la muestra entre los grupos análisis, lo que genera un sesgo dentro del análisis de esta variable. Este estudio muestra y resalta la importancia de generar estudios similares en países de medianos y bajos ingresos que 
permitan su comparación. Además, permite ver la necesidad de generar políticas de salud con base a los resultados observados para cada población.

López-Hernández Daniel Doctor en Ciencias. Director, Centro de Investigaciones y de Educación Continua, CENINVEC. Investigador, Instituto de Seguridad y Servicios Sociales de la Trabajadores del Estado, ISSSTE (Ciudad de México, México).

\section{Referencias}

1. Nazzal C, Corbalán R, Díaz C, Sepúlveda P, Schacht E, Grupo GEMI. Efecto del nivel educacional en la sobrevida

Los autores aludidos ofrecieron la siguiente réplica:

Asociación entre el nivel de educación y la sobreviva después de presentar un infarto agudo de miocardio. Réplica

\section{Association between educational level and survival after acute myocardial infarction. Reply}

En consideración a la carta enviada por el Dr. Daniel López-Hernández me permito informar:

El Dr. López considera que el no especificar el rango de edad de la muestra pudiese estar afectando los resultados encontrados. Tal como menciona, la edad es un factor pronóstico, la edad media de la muestra fue $63,1 \pm 13$ años, pero los resultados que se muestran en nuestro artículo, tanto para establecer las asociaciones descritas de mayor riesgo con menor nivel educacional, como la proporción de muerte al interior de las distintas categorías de educación, han sido ajustadas por edad.

En relación al empleo de códigos CIE-10 para la identificación de los casos, ésta no se empleó, sino que se ingresaron los pacientes que resultaron elegibles según los criterios clínicos del registro chileno de infarto de miocardio, Registro GEMI, que consideran elevación enzimática, cambios electrocardiográficos característicos y dolor precordial. Al ser este un registro prospectivo, los casos de infarto incluidos son casos incidentes, y como se describe en los métodos, corresponden a los pacientes que consultaron y fueron hospitalizados en los hospitales del estudio en el período descrito. En aquellos pacientes que presentaron más de un episodio de IAM durante el período del estudio, se consideró, para efecto de evaluar la sobrevida, el primer evento.

En lo que respecta a causa de muerte total como elección de evento primario, esta decisión se basa en la posterior a un infarto agudo de miocardio: Registro Chileno de Infarto de Miocardio, GEMI 2009-2012. Rev Med Chile 2015; 143: 825-33.

2. Igland J, Vollset SE, Nygård OK, Sulo G, Ebbing M, Tell GS. Educational inequalities in acute myocardial infarction incidence in Norway: a nationwide cohort study. PLoS One 2014; 9 (9): e106898.

Declaración de Conflicto de interés

El autor declara no tener conflicto de interés

Correspondencia a:

Dr. Daniel López Hernández

2003dlopez@gmail.com

limitación que presentan los certificados de defunción para establecer con certeza la causa de muerte (fuente de información empleada en este estudio), especialmente en población de edad avanzada y muertes por causa cardiovascular. Coincidimos que es de interés conocer la sobrevida por causas específicas, pero reconocemos que la clasificación de la causa de muerte basada solo en los certificados de defunción, presenta limitaciones.

La clasificación de educación básica y media se basa en los años de estudios cursados. Se consideró como nivel básico menos de 8 años completos (en Chile la educación básica cursa de primero a octavo básico), y como nivel medio entre 8 y 12 años de estudio (la educación media incluye de primero a cuarto medio).

Respecto del análisis de muerte por grupo de educación, el Dr. López-Hernandez advierte que el mayor número de muertes se produce en los grupos de educación básica y media, lo cual es esperable, dado que son los grupos con mayor tamaño. Sin embargo, lo que interesaba estudiar es la proporción de muertes (evento en estudio) respecto el total de cada categoría de educación (exposición en estudio), no respecto del total de muertes, como se muestra en las Figuras la y $1 \mathrm{~b}$.

Otro aspecto que discute es en relación al uso de fármacos. En este análisis, la variable uso se refiere al empleo de ese fármaco, independientemente del uso en paralelo de otros fármacos. Sabemos que en este grupo de pacientes la terapia combinada (IECA, estatinas, aspirina y beta bloqueadores) es altamente utilizada, dado que es una recomendación de la actual guía clínica para IAM. El análisis por combinación de fármacos no se realizó y el efecto protector que se muestra es en relación al empleo de cada fármaco en particular, independientemente de las posibles combinaciones. El incluir los fármacos responde a la necesidad de ajustar 